\title{
Homocoupling and Heterocoupling of Grignard Perfluorobenzene Reagents via Aryne Intermediates: A DFT Study
}

Lavrenty G. Gutsev, ${ }^{,}+$Gennady L. Gutsev ${ }^{\ddagger}$, Katharine Moore Tibbetts§ ${ }^{\S}$ and Puru Jena, ${ }^{\dagger}$

${ }^{\dagger}$ Department of Physics, Virginia Commonwealth University, Richmond, VA 23284, USA

‡ Department of Physics, Florida A\&M University, Tallahassee, Florida 32307, USA

$\S$ Department of Chemistry, Virginia Commonwealth University, Richmond, VA 23284, USA 
Table S1. Changes in free energy at $298.15 \mathrm{~K} \Delta G(298 \mathrm{~K})$ and solvated free energy $\Delta G^{T H F}(298 \mathrm{~K})$ for the homocoupling mechanism shown in Fig. 5 at the BPW91, B3LYP, CAM-B3LYP and 6 B97XD levels of theory. All values are in $\mathrm{kcal} / \mathrm{mol}$.

\begin{tabular}{|c|c|c|c|c|}
\hline \multicolumn{5}{|c|}{ Vacuum } \\
\hline & BPW91 & B3LYP & CAM-B3LYP & WB97XD \\
\hline$\left(\mathrm{C}_{6} \mathrm{~F}_{5}\right)-\mathrm{Mg}-\mathrm{F}+\left(\mathrm{C}_{6} \mathrm{~F}_{5}\right)-\mathrm{Mg}-\mathrm{F} \rightarrow \mathrm{Mg}_{2}\left(\mathrm{C}_{12} \mathrm{~F}_{12}\right) \mathrm{LM1}$ & -3.6 & -8.6 & -12.2 & -13.9 \\
\hline$\left(\mathrm{C}_{6} \mathrm{~F}_{5}\right)-\mathrm{Mg}-\mathrm{F}+\left(\mathrm{C}_{6} \mathrm{~F}_{5}\right)-\mathrm{Mg}-\mathrm{F}-\mathrm{Mg}_{2}\left(\mathrm{C}_{12} \mathrm{~F}_{12}\right) \mathrm{LM} 2$ & -26.6 & -32.1 & -35.2 & -33.4 \\
\hline$\left(\mathrm{C}_{6} \mathrm{~F}_{5}\right)-\mathrm{Mg}-\mathrm{F}+\left(\mathrm{C}_{6} \mathrm{~F}_{5}\right)-\mathrm{Mg}-\mathrm{F} \rightarrow \mathrm{Mg}_{2}\left(\mathrm{C}_{12} \mathrm{~F}_{12}\right) \mathrm{LM} 3$ & -42.7 & -47.2 & -50.2 & -49.8 \\
\hline $\operatorname{Mg} 2\left(C_{12} F_{12}\right)$ LM1 $\rightarrow \operatorname{Mg}_{2}\left(C_{12} F_{12}\right)$ LM4 & -23.5 & -25.4 & -26.1 & -33.4 \\
\hline $\operatorname{Mg} 2\left(C_{12} F_{12}\right)$ LM2 $->M g_{2}\left(C_{12} F_{12}\right)$ LM4 & -0.5 & -1.9 & -3.1 & -3.8 \\
\hline $\operatorname{Mg} 2\left(C_{12} F_{12}\right) L M 4->M g_{2}\left(C_{12} F_{12}\right)$ LM5 & 3.7 & 6.2 & 6.0 & 4.8 \\
\hline$\left(\mathrm{C}_{6} \mathrm{~F}_{5}\right)-\mathrm{Mg}-\mathrm{F}+\left(\mathrm{C}_{6} \mathrm{~F}_{5}\right)-\mathrm{Mg}-\mathrm{F} \rightarrow \mathrm{Mg}_{2} \mathrm{~F}_{3}\left(\mathrm{C}_{12} \mathrm{~F}_{9}\right) \mathrm{P1}$ & -69.6 & -75.3 & -79.9 & -86.2 \\
\hline$\left(\mathrm{C}_{6} \mathrm{~F}_{5}\right)-\mathrm{Mg}-\mathrm{F}+\left(\mathrm{C}_{6} \mathrm{~F}_{5}\right)-\mathrm{Mg}-\mathrm{F}-\mathrm{Mg}_{2} \mathrm{~F}_{3}\left(\mathrm{C}_{12} \mathrm{~F}_{9}\right) \mathrm{P} 2$ & -83.2 & -91.0 & -96.1 & -95.2 \\
\hline \multicolumn{5}{|c|}{ Activation Barriers } \\
\hline $\operatorname{Mg} 2\left(C_{12} F_{12}\right)$ LM4 $->\operatorname{Mg} 2\left(C_{12} F_{12}\right)$ TS1 & 5.6 & 5.8 & 6.7 & 5.8 \\
\hline $\operatorname{Mg} 2\left(C_{12} F_{12}\right)$ LM5 $->\operatorname{Mg} 2\left(C_{12} F_{12}\right)$ TS2 & 28.1 & 29.1 & 34.3 & 33.8 \\
\hline $\operatorname{Mg} 2\left(C_{12} F_{12}\right)$ LM2 $\rightarrow \operatorname{Mg} 2\left(C_{12} F_{12}\right)$ TS4 & 18.1 & 21.9 & 25.6 & 24.6 \\
\hline $\operatorname{Mg} 2\left(C_{12} F_{12}\right)$ LM3 $\rightarrow \operatorname{Mg} 2\left(C_{12} F_{12}\right)$ TS3 & 32.2 & 32.8 & 37.5 & 37.7 \\
\hline \multicolumn{5}{|c|}{ SCRF Solvent $=$ TetraHydroFuran } \\
\hline & BPW91 & B3LYP & CAM-B3LYP & wB97XD \\
\hline$\left(\mathrm{C}_{6} \mathrm{~F}_{5}\right)-\mathrm{Mg}-\mathrm{F}+\left(\mathrm{C}_{6} \mathrm{~F}_{5}\right)-\mathrm{Mg}-\mathrm{F} \rightarrow \mathrm{Mg}_{2}\left(\mathrm{C}_{12} \mathrm{~F}_{12}\right) \mathrm{LM1}$ & -42.2 & -46.9 & -49.0 & -55.6 \\
\hline$\left(\mathrm{C}_{6} \mathrm{~F}_{5}\right)-\mathrm{Mg}-\mathrm{F}+\left(\mathrm{C}_{6} \mathrm{~F}_{5}\right)-\mathrm{Mg}-\mathrm{F}->\mathrm{Mg}_{2}\left(\mathrm{C}_{12} \mathrm{~F}_{12}\right)$ LM2 & -61.4 & -66.9 & -69.8 & -71.5 \\
\hline$\left(\mathrm{C}_{6} \mathrm{~F}_{5}\right)-\mathrm{Mg}-\mathrm{F}+\left(\mathrm{C}_{6} \mathrm{~F}_{5}\right)-\mathrm{Mg}-\mathrm{F}-\mathrm{Mg}_{2}\left(\mathrm{C}_{12} \mathrm{~F}_{12}\right) \mathrm{LM} 3$ & -74.1 & -78.9 & -81.5 & -83.0 \\
\hline $\mathrm{Mg}_{2}\left(\mathrm{C}_{12} \mathrm{~F}_{12}\right)$ LM1 $->\mathrm{Mg}_{2}\left(\mathrm{C}_{12} \mathrm{~F}_{12}\right)$ LM4 & -14.8 & -19.4 & -19.8 & -14.7 \\
\hline $\mathrm{Mg}_{2}\left(\mathrm{C}_{12} \mathrm{~F}_{12}\right) \mathbf{L M} 2->\mathrm{Mg}_{2}\left(\mathrm{C}_{12} \mathrm{~F}_{12}\right)$ LM4 & -0.5 & -1.9 & -3.1 & -3.8 \\
\hline $\mathrm{Mg}_{2}\left(\mathrm{C}_{12} \mathrm{~F}_{12}\right)$ LM4 -> Mg$\left(\mathrm{C}_{12} \mathrm{~F}_{12}\right)$ LM5 & 4.4 & 0.6 & 1.0 & 1.26 \\
\hline$\left(\mathrm{C}_{6} \mathrm{~F}_{5}\right)-\mathrm{Mg}-\mathrm{F}+\left(\mathrm{C}_{6} \mathrm{~F}_{5}\right)-\mathrm{Mg}-\mathrm{F}->\mathrm{Mg}_{2} \mathrm{~F}_{3}\left(\mathrm{C}_{12} \mathrm{~F}_{9}\right) \mathrm{P1}$ & -100.3 & -107.2 & -111.1 & -117.0 \\
\hline$\left(\mathrm{C}_{6} \mathrm{~F}_{5}\right)-\mathrm{Mg}-\mathrm{F}+\left(\mathrm{C}_{6} \mathrm{~F}_{5}\right)-\mathrm{Mg}-\mathrm{F} \rightarrow \mathrm{Mg}_{2} \mathrm{~F}_{3}\left(\mathrm{C}_{12} \mathrm{~F}_{9}\right) \mathrm{P} 2$ & -114.3 & -122.4 & -126.6 & -129.9 \\
\hline \multicolumn{5}{|c|}{ Activation Barriers } \\
\hline $\mathrm{Mg}_{2}\left(\mathrm{C}_{12} \mathrm{~F}_{12}\right)$ LM4 -> $\mathrm{Mg}_{2}\left(\mathrm{C}_{12} \mathrm{~F}_{12}\right)$ TS1 & -2.2 & 1.5 & 1.3 & 0.2 \\
\hline $\mathrm{Mg}_{2}\left(\mathrm{C}_{12} \mathrm{~F}_{12}\right)$ LM5 -> $\mathrm{Mg}_{2}\left(\mathrm{C}_{12} \mathrm{~F}_{12}\right)$ TS2 & 25.6 & 30.9 & 35.0 & 36.6 \\
\hline $\operatorname{Mg} 2\left(C_{12} F_{12}\right) \operatorname{LM} 2->\operatorname{Mg} 2\left(C_{12} F_{12}\right)$ TS4 & 27.8 & 27.8 & 34.8 & 36.1 \\
\hline $\operatorname{Mg} 2\left(C_{12} F_{12}\right)$ LM3 $\rightarrow \operatorname{Mg} 2\left(C_{12} F_{12}\right)$ TS3 & 43.8 & 42.8 & 48.5 & 49.0 \\
\hline
\end{tabular}



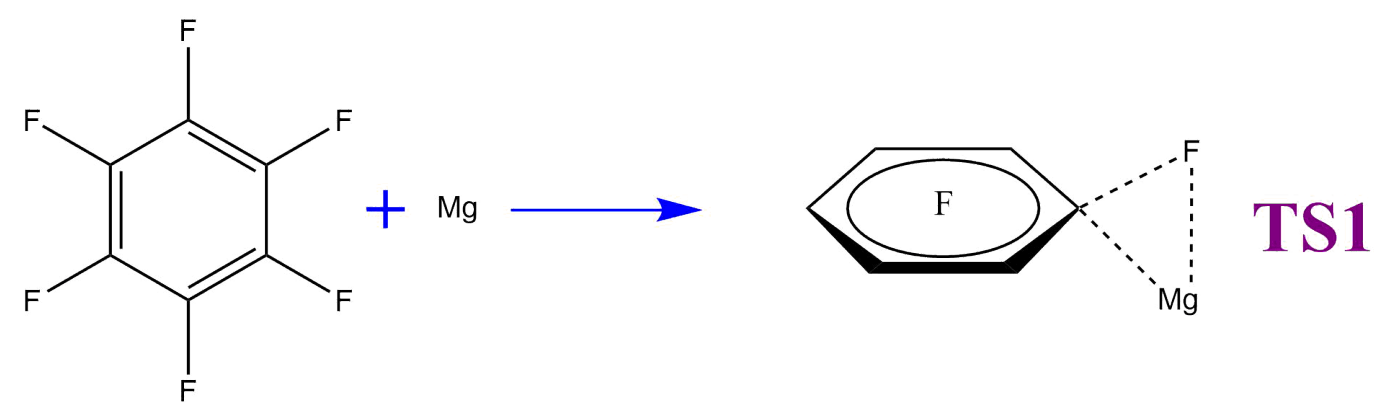

\section{BPW91 PBE0 B3LYP CAM-B3LYP wB97XD CBS-4M

$\begin{array}{ccccccc}\Delta \mathrm{G}_{298}^{*} & \mathbf{1 5 . 0} & \mathbf{2 1 . 3} & \mathbf{2 0 . 5} & 25.4 & 26.1 & 25.1 \\ \Delta \mathrm{G}_{298}^{* T H F} & \mathbf{6 . 6} & \mathbf{1 0 . 6} & 11.2 & 15.3 & 14.6 & 16.3\end{array}$

Figure S1. Comparison of the energy of metalation computed by a variety of methods. 

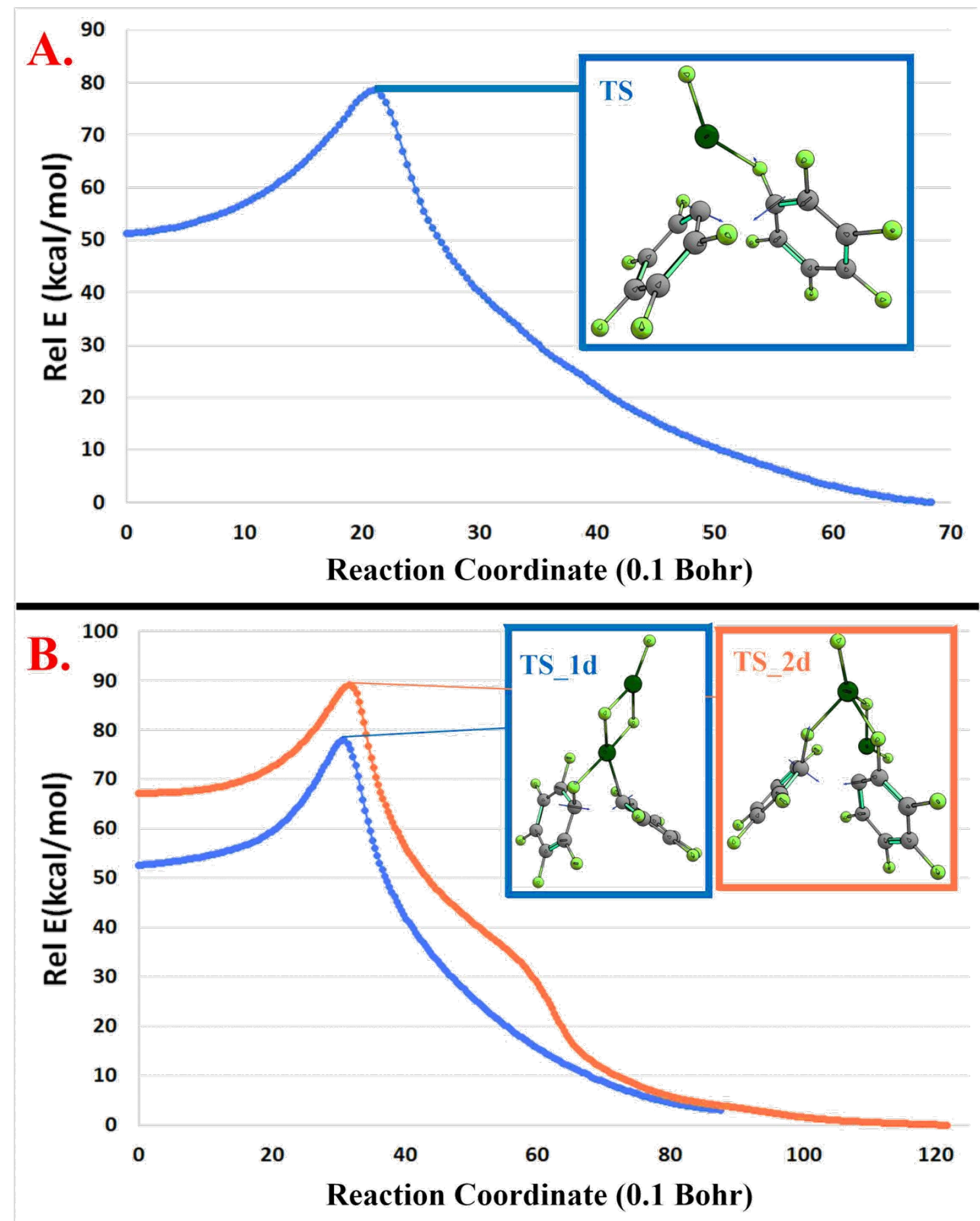

$\rightarrow$ Single-Bridged (BPW91) $\rightarrow$ Bidentate (BPW91)

Figure S2. Internal reaction coordinates versus the relative energy for heterocoupling of $\left(A_{1}\right)\left(C_{6} F_{5}\right)-M g$ $-\mathrm{F}+\left(\mathrm{C}_{6} \mathrm{~F}_{6}\right)\left(\mathrm{SCRF}\right.$ THF) and (B.) $\left(\mathrm{C}_{6} \mathrm{~F}_{5}\right)-\mathrm{Mg}_{2} \mathrm{~F}_{3}+\left(\mathrm{C}_{6} \mathrm{~F}_{6}\right)$ (vacuum) where the blue line is single-bridged and orange is bidentate. 


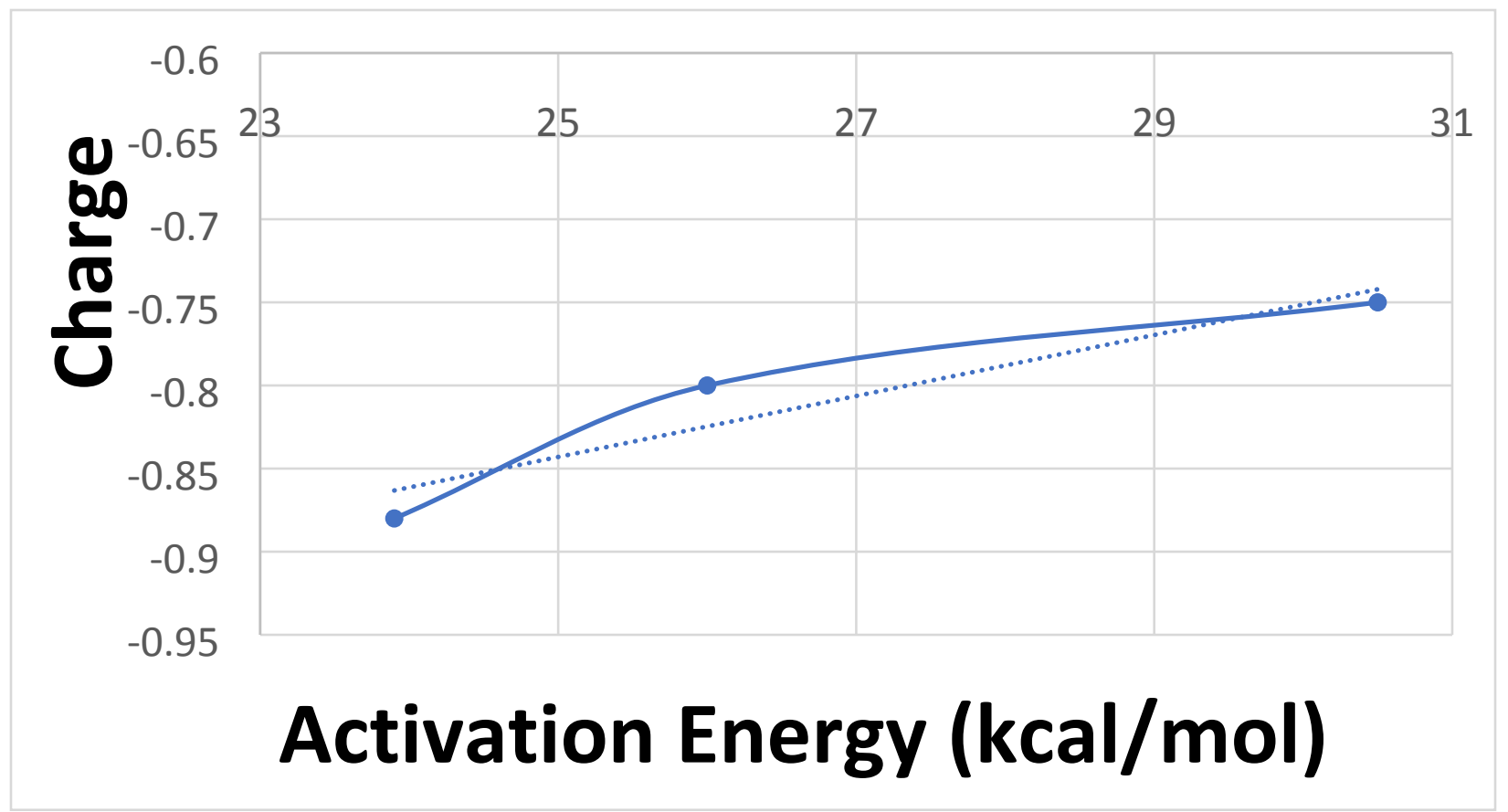

Figure S3. The Charge of the Nucleophilic Carbon versus the activation barrier of the Grignard heterocoupling in vacuum. 


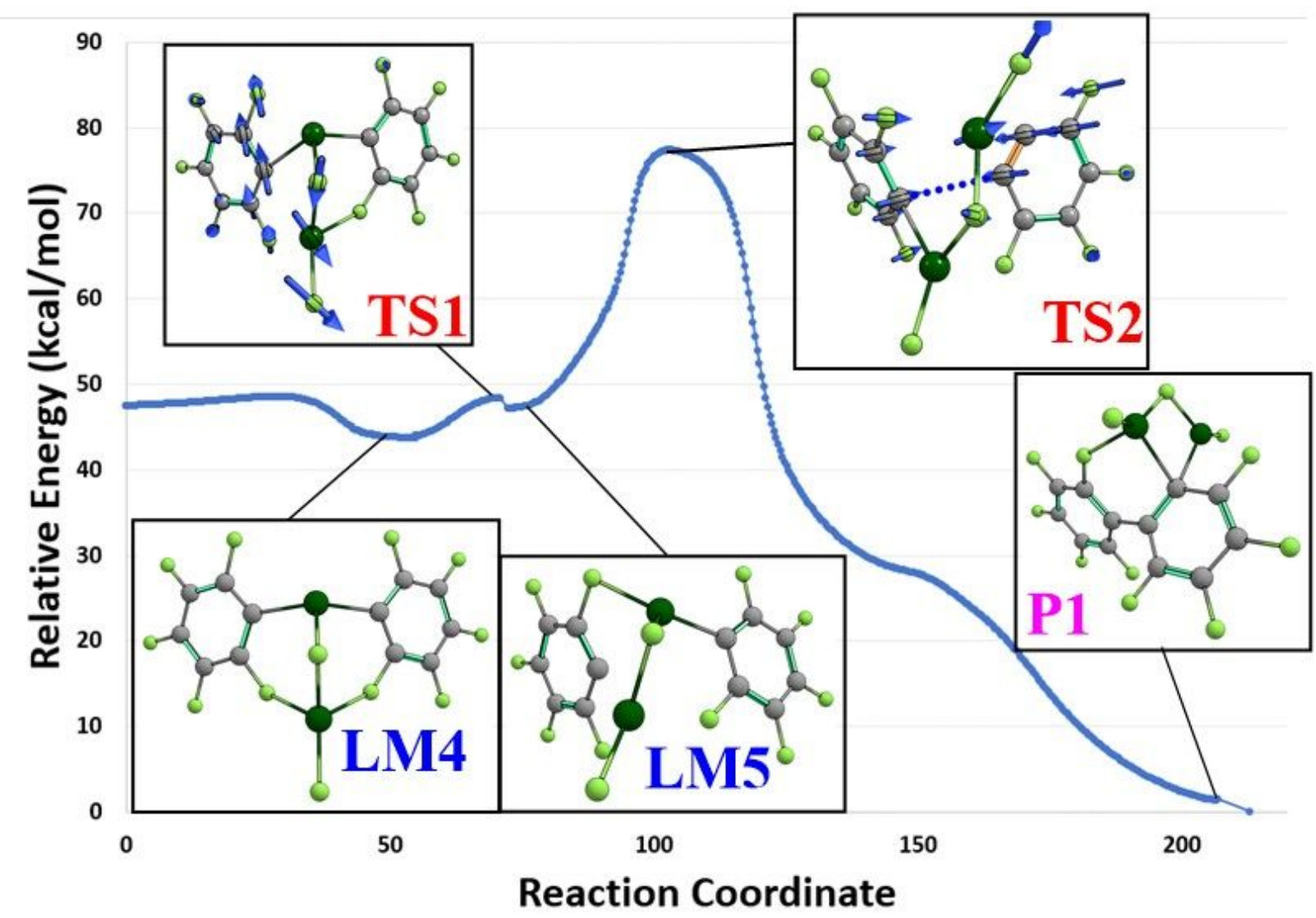

Figure S4. Internal reaction coordinates versus the relative energy for $\left(C_{6} F_{5}\right)-M g-F+\left(C_{6} F_{5}\right)-M g-F$ homocoupling Pathway 1. In particular, the BPW91 curve is the combination of multiple IRC scans.

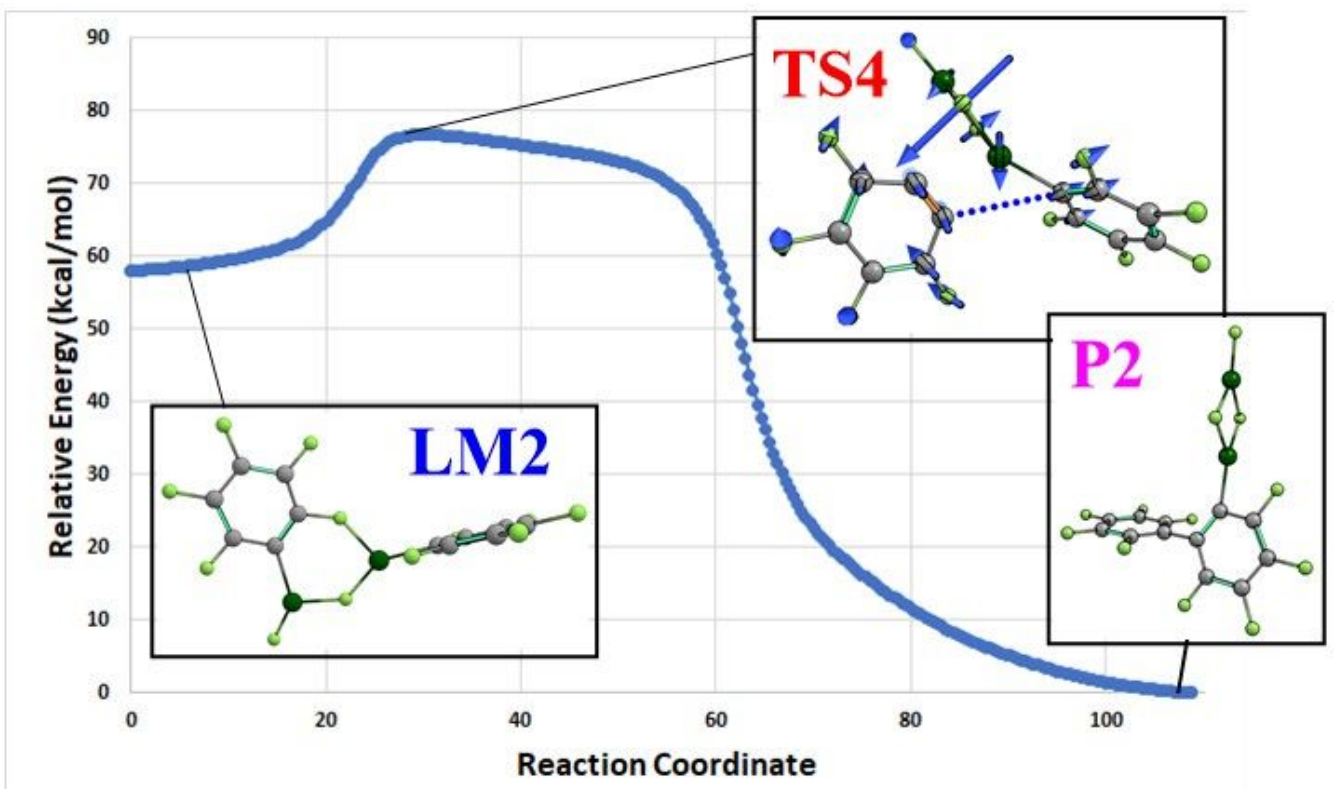

Figure S5. The internal reaction coordinate versus the relative energy for $\left(C_{6} F_{5}\right)-M g-F+\left(C_{6} F_{5}\right)-M g-F$ homocoupling in Pathway 2. 


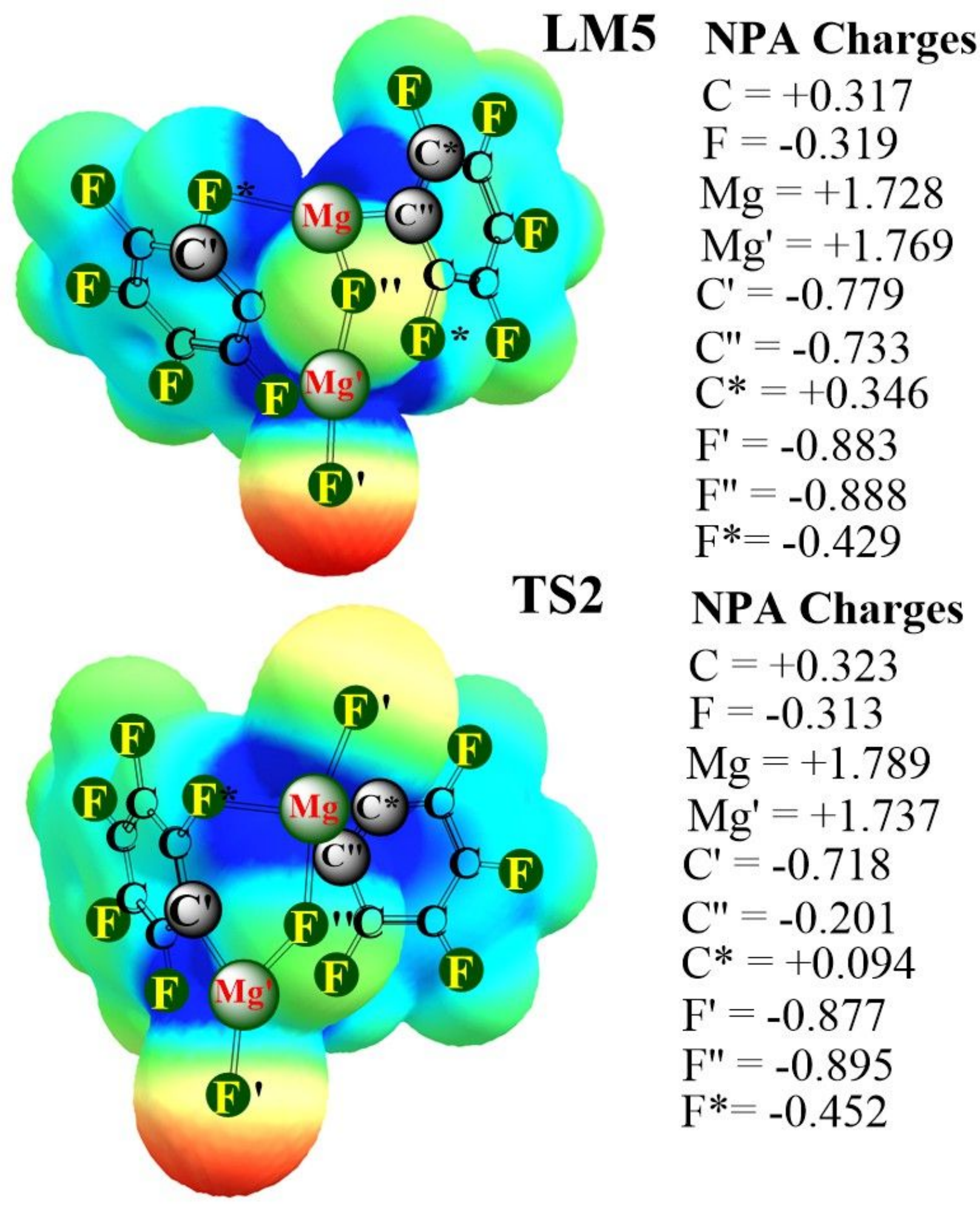

Figure S6. Electrostatic Potential surface (ESP) and NPA charges of IM2 and TS2. The nucleophilic carbons are represented by gray circles to emphasize them. 

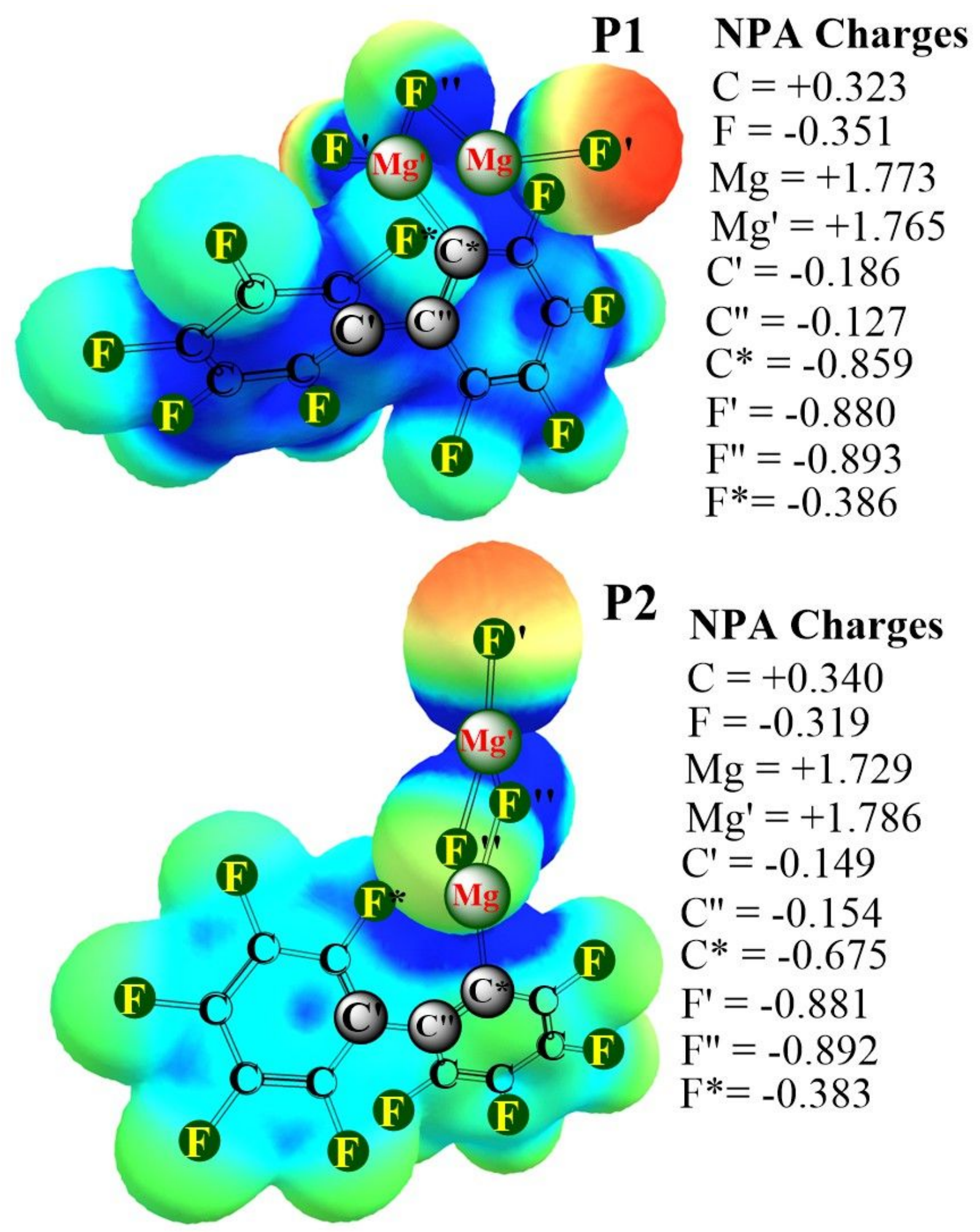

Figure S7. Electrostatic Potential surface (ESP) and NPA charges of P1 and P2. The nucleophilic carbons are represented by gray circles to emphasize them. 


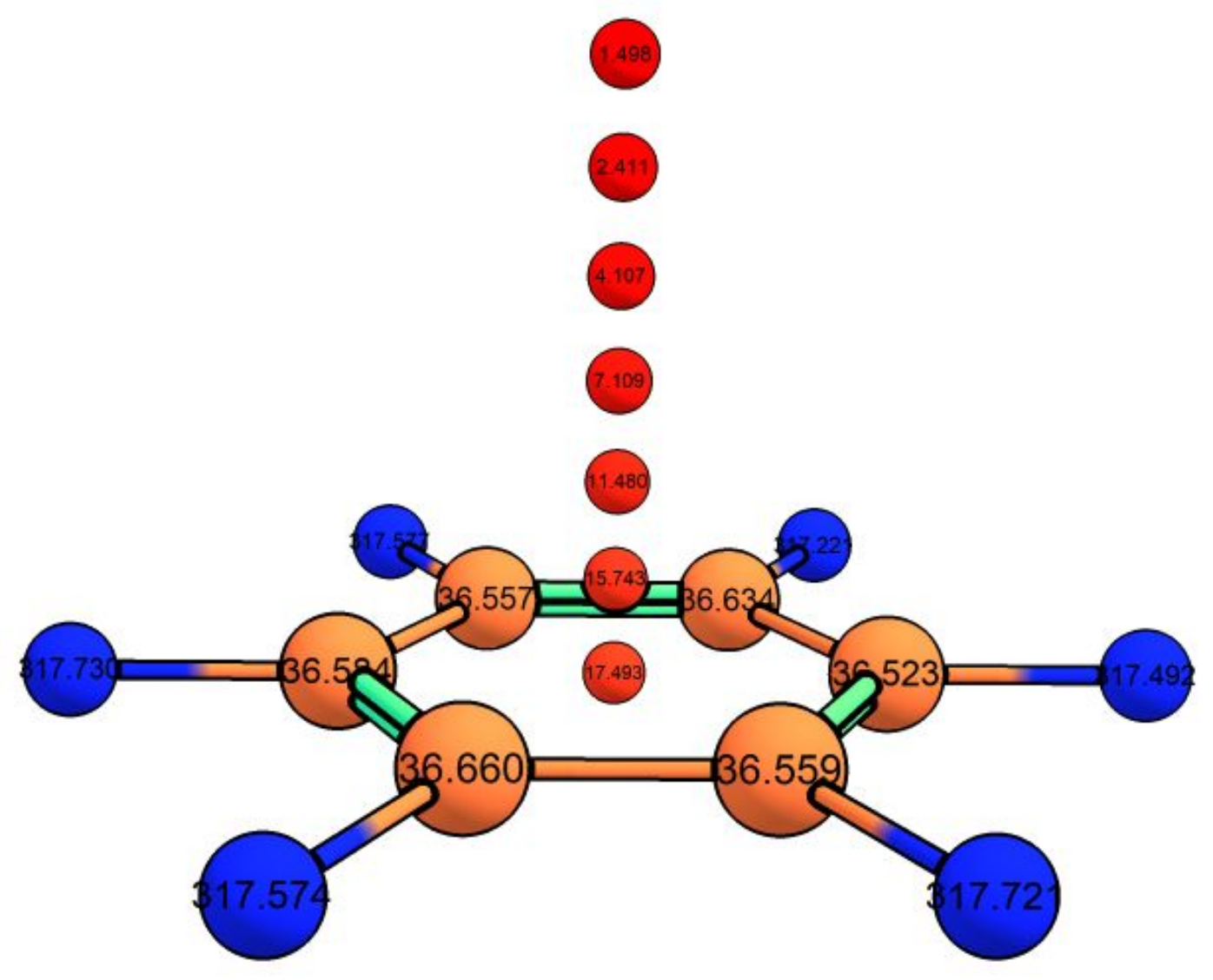

Figure S8. NICS values of hexafluorobenzene in increments of 0.5 Angstrom. 


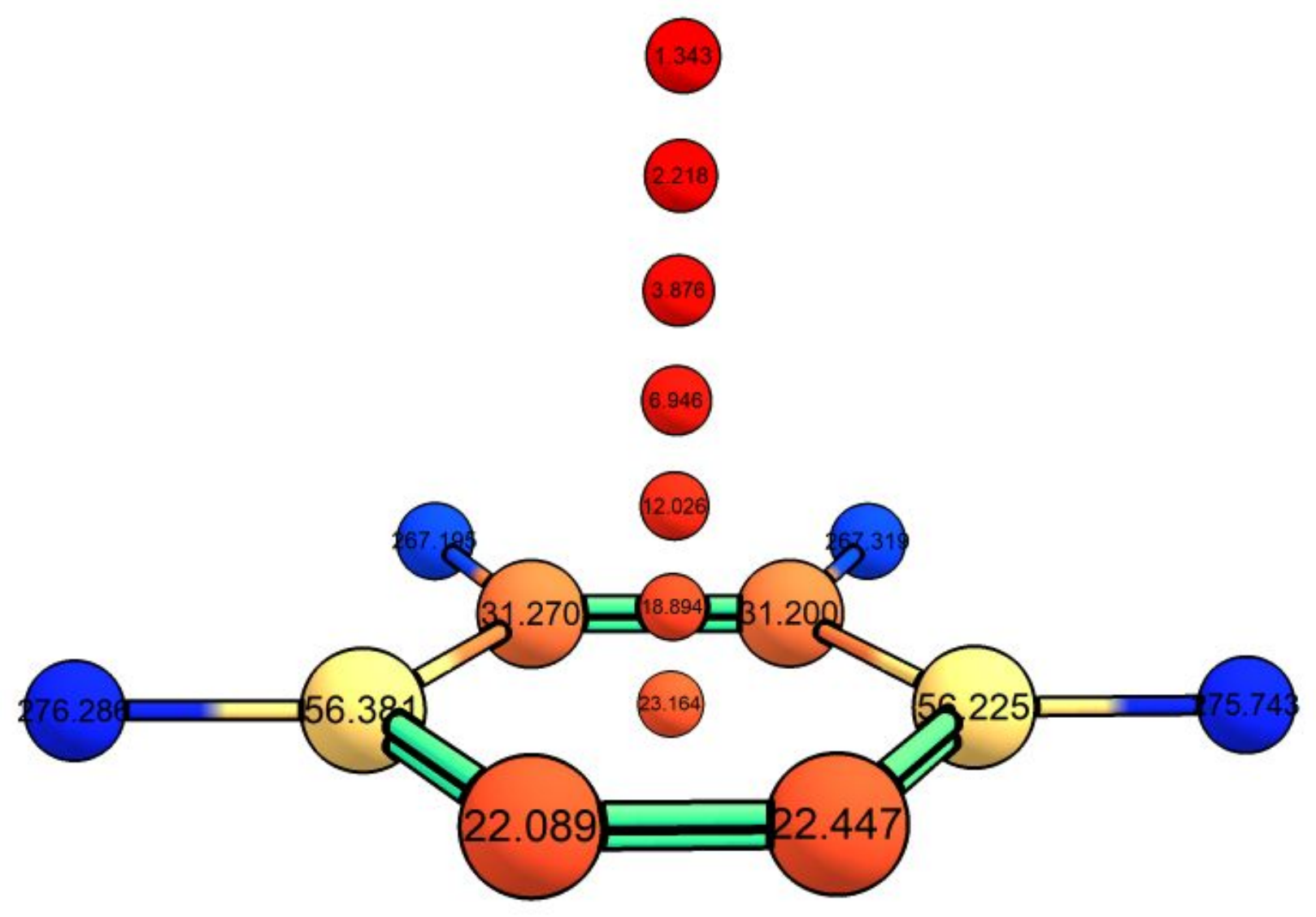

Figure S9. NICS values of tetrafluoro-benzyne in increments of 0.5 Angstrom. 


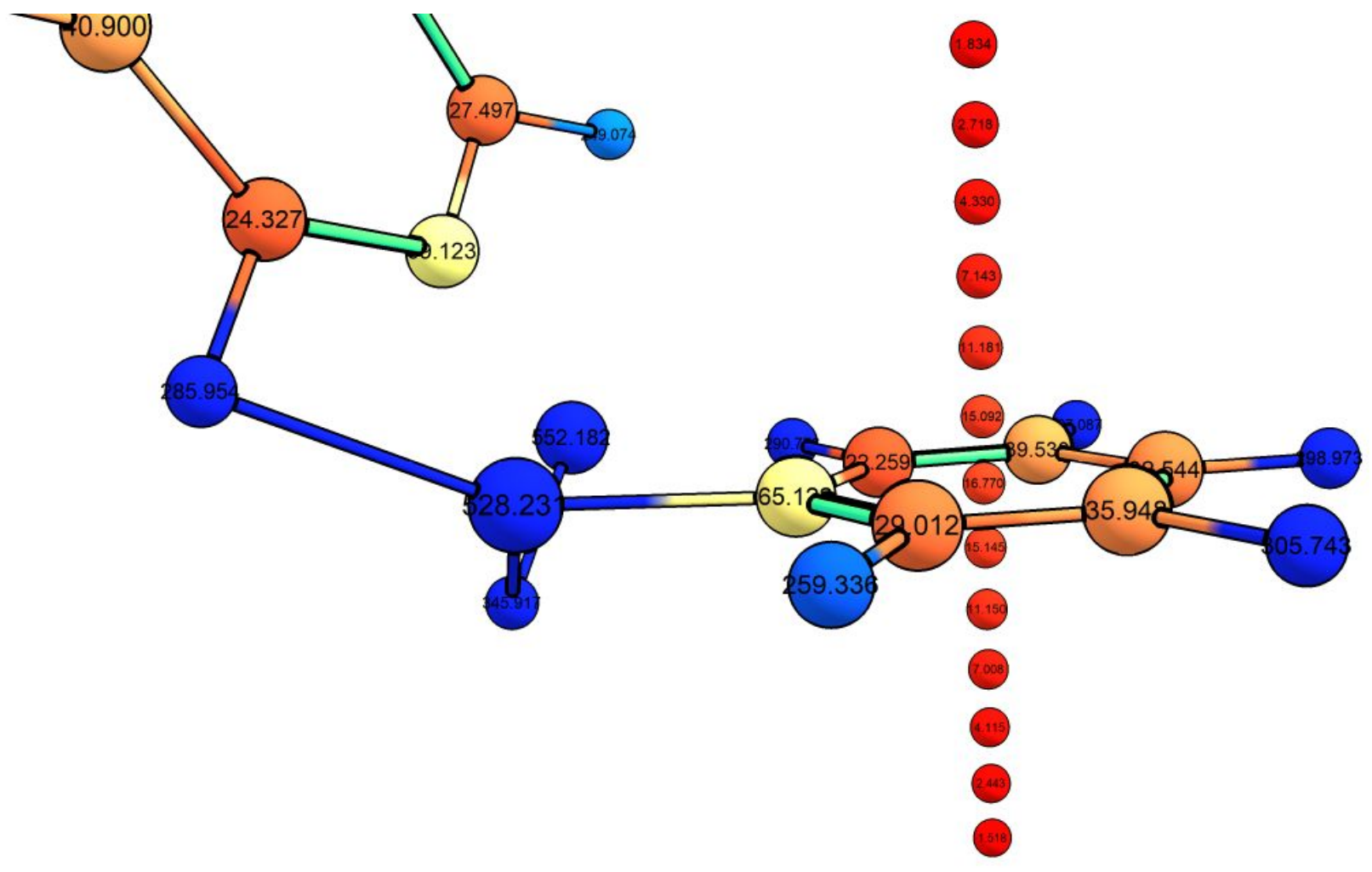

Figure S10. NICS values of LM5 on the $C^{\prime \prime} C^{*}$ (receiving) side in increments of 0.5 Angstrom. 


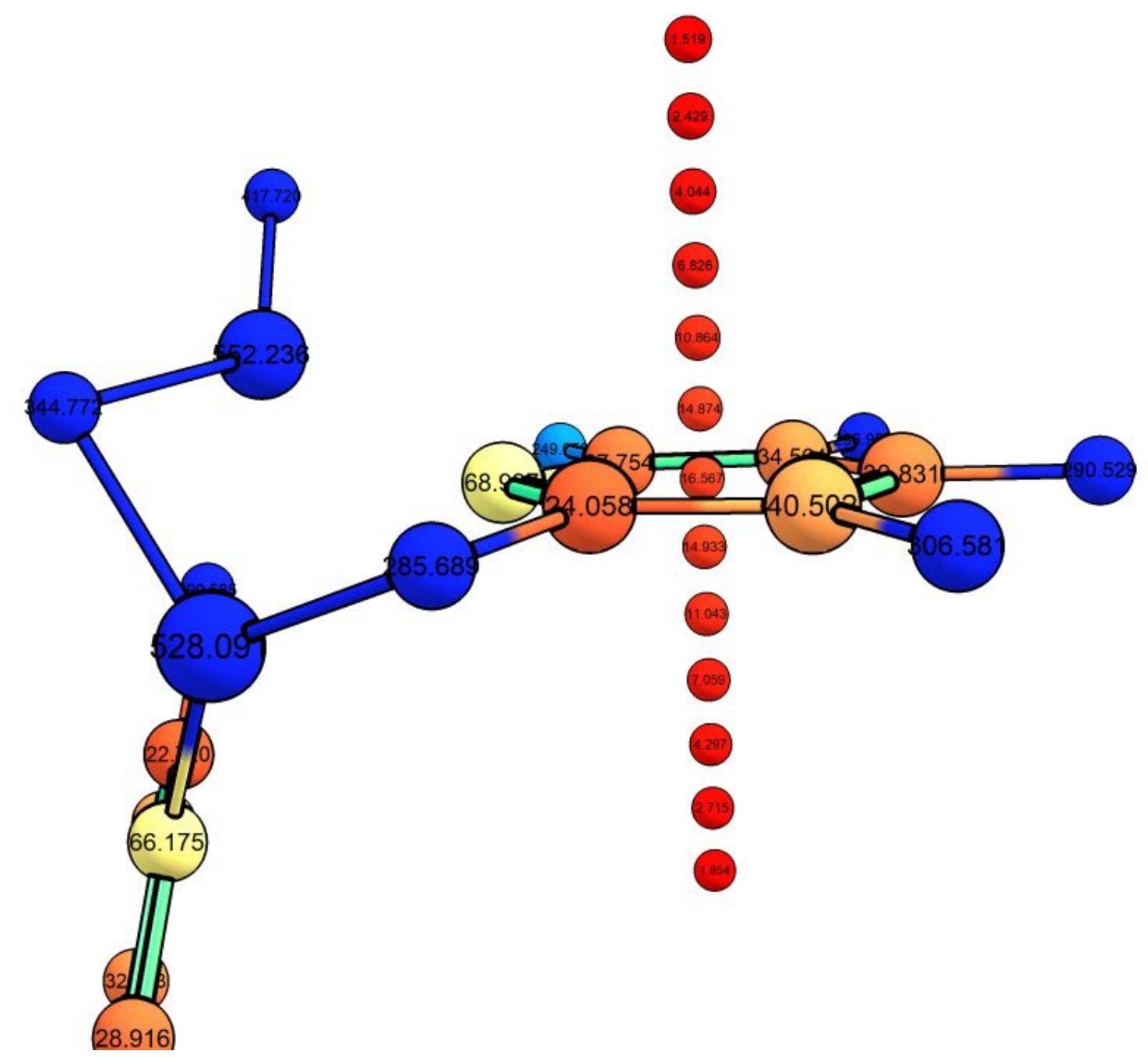

Figure S11. NICS values of LM5 on the C' (donating) side in increments of 0.5 Angstrom. 


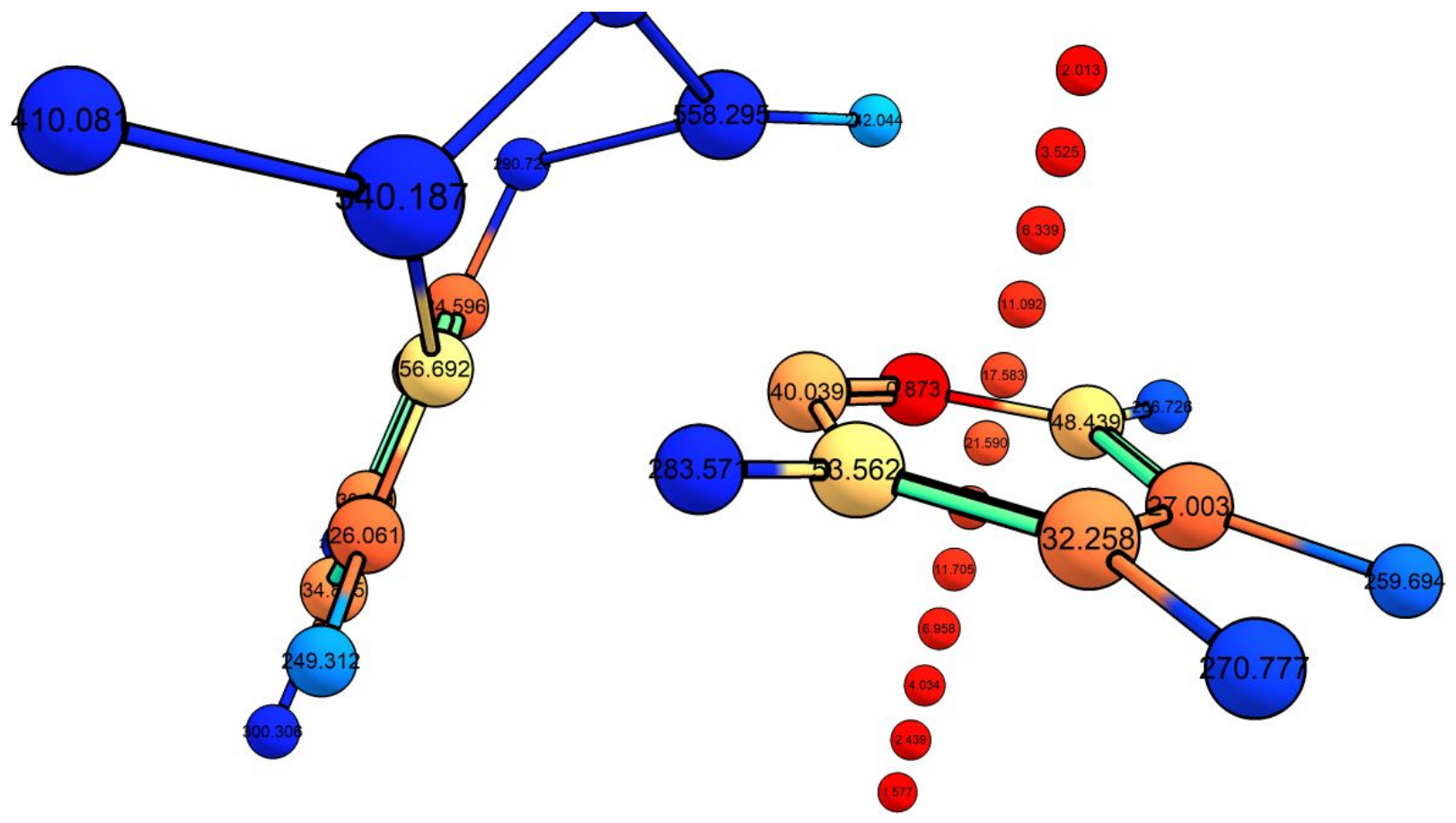

Figure S12. NICS values of TS2 on the C" C* (receiving) side in increments of 0.5 Angstrom. 


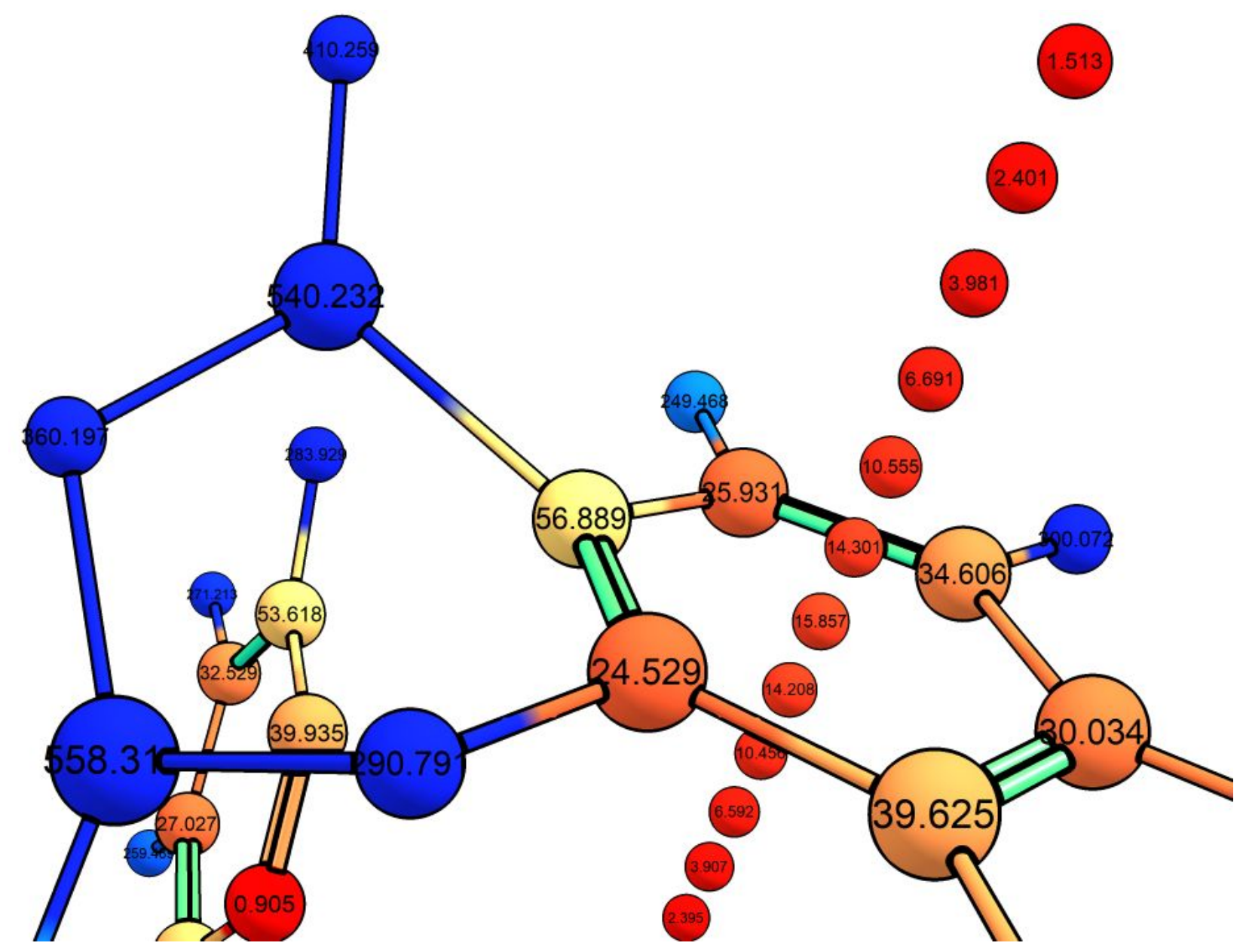

Figure S13. NICS values of TS2 on the $C^{\prime}$ (donating) side in increments of 0.5 Angstrom. 


\section{Mg(atom)}

$E(w B 97 X D):-200.0579712$

\section{Hexaflurobenzene}

$\begin{array}{lrrr}C & -0.222414000 & 0.000000000 & -2.597178000 \\ \text { C } & -1.370591000 & -0.352247000 & -1.903756000 \\ \text { C } & 0.925763000 & 0.352247000 & -1.903756000 \\ \text { C } & -1.370591000 & -0.352247000 & -0.516934000 \\ \text { C } & 0.925763000 & 0.352247000 & -0.516934000 \\ \text { C } & -0.222414000 & 0.000000000 & 0.176488000 \\ \text { F } & -2.468188000 & -0.689031000 & -2.566536000 \\ \text { F } & -2.468188000 & -0.689031000 & 0.145846000 \\ \text { F } & -0.222414000 & 0.000000000 & -3.922855000 \\ \text { F } & -0.222414000 & 0.000000000 & 1.502165000 \\ \text { F } & 2.023361000 & 0.689031000 & 0.145846000 \\ \text { F } & 2.023361000 & 0.689031000 & -2.566536000 \\ \text { E(WB97XD):-827.5939522 } & \end{array}$




$$
\left(C_{6} F_{5}\right) M g F(T S)
$$

$\begin{array}{llll}C & -1.826604000 & -0.085372000 & -0.288833000 \\ \text { C } & -1.325764000 & -1.290479000 & 0.189010000 \\ \text { C } & -0.977438000 & 1.000745000 & -0.464396000 \\ \text { F } & -2.137486000 & -2.333510000 & 0.320314000 \\ \text { F } & -1.457703000 & 2.137746000 & -0.954900000 \\ \text { C } & 0.009268000 & -1.391869000 & 0.530644000 \\ \text { C } & 0.356440000 & 0.891873000 & -0.120581000 \\ \text { F } & 0.493827000 & -2.559710000 & 0.955417000 \\ \text { F } & 1.175595000 & 1.924796000 & -0.323537000 \\ \text { C } & 0.870205000 & -0.322831000 & 0.316062000 \\ \text { F } & 2.030889000 & -0.189955000 & 1.400915000 \\ \text { F } & -3.114559000 & 0.025739000 & -0.585671000 \\ \text { Mg } & 3.162715000 & -0.795048000 & -0.121356000 \\ \text { E(wB97XD) = -1027.61987178 } & \end{array}$




$$
\left(C_{6} F_{5}\right) M g F-T H F(T S)
$$

$$
\begin{aligned}
& \text { C } \quad-1.872595000 \quad-1.604479000 \quad-0.647379000 \\
& \text { C } \quad-0.730445000 \quad-1.576006000 \quad 0.163773000 \\
& \text { C } \quad-1.738664000 \quad-1.518152000 \quad-2.040153000 \\
& \text { F } \quad-0.856725000 \quad-1.713581000 \quad 1.499854000 \\
& \text { F } \quad-2.836993000 \quad-1.595125000 \quad-2.818869000 \\
& \text { C } \quad 0.522909000 \quad-1.396416000 \quad-0.411474000 \\
& \text { C } \quad-0.482499000 \quad-1.339973000 \quad-2.611546000 \\
& \begin{array}{llll}
\text { F } & 1.635045000 & -1.455857000 & 0.370521000
\end{array} \\
& \text { F } \quad-0.355026000 \quad-1.322811000 \quad-3.958601000 \\
& \begin{array}{llll}
\text { C } & 0.670854000 & -1.358365000 & -1.808055000
\end{array} \\
& \text { F } \quad 1.850623000 \quad-0.717880000 \quad-2.327043000 \\
& \text { F } \quad-3.096142000 \quad-1.692436000 \quad-0.089918000 \\
& \begin{array}{llll}
\text { Mg } & 2.468818000 & -3.137388000 & -2.597301000
\end{array} \\
& \begin{array}{llll}
\text { C } & 5.713886000 & -0.345336000 & -2.508950000
\end{array} \\
& \text { C } \quad 6.216443000 \quad-1.266428000 \quad-3.658183000 \\
& \text { C } \quad 5.630421000 \quad-2.652719000 \quad-3.323518000 \\
& \text { O } \quad 4.555137000 \quad-2.400348000 \quad-2.373729000 \\
& \text { C } \quad 5.028265000 \quad-1.307908000 \quad-1.532444000 \\
& \begin{array}{llll}
\text { H } & 4.988309000 & 0.386334000 & -2.888447000
\end{array} \\
& \begin{array}{llll}
\text { H } & 7.312955000 & -1.308749000 & -3.707363000
\end{array} \\
& \text { H } \quad 5.186150000 \quad-3.182344000 \quad-4.176718000 \\
& \text { H } \quad 4.153922000 \quad-0.897218000 \quad-1.016629000 \\
& \text { H } \quad 6.371762000 \quad-3.310557000 \quad-2.841756000 \\
& \text { H } \quad 5.856708000 \quad-0.908389000 \quad-4.631832000 \\
& \begin{array}{llll}
H & 6.528708000 & 0.210424000 & -2.025378000
\end{array} \\
& \begin{array}{llll}
\text { H } & 5.730833000 & -1.720860000 & -0.789403000
\end{array} \\
& E(B P W 91)=-1260.30778180
\end{aligned}
$$


$\left(C_{6} F_{5}\right) M g F-T H F(T S 2)$
C $\quad-1.813887000$
$-1.615409000$
$-0.711471000$
C $\quad-0.681139000-1.588235000$
0.111998000
C -1.664060000
F $\quad-0.821566000$
$\mathrm{F}$
$-2.755889000$
$-1.733346000$
$-2.100822000$
C
C
C -0.403489000
$-1.600674000-2.891495000$
$\mathrm{F}$
1.68129500
$-1.352491000$
$-0.449567000$
F
F
C
$-0.264028000$
$-1.469029000$
$-2.659540000$
$\mathrm{F}$
F
$\mathrm{F}$
$\mathrm{M}$

$$
\mathrm{C}
$$
C
$\begin{array}{llll}\text { C } & 5.658928000 & -2.666904000 & -3.290530000\end{array}$
$\begin{array}{llll}\text { O } & 4.607837000 & -2.464315000 & -2.303802000\end{array}$
$\begin{array}{llll}\text { C } & 5.056835000 & -1.348333000 & -1.481809000\end{array}$
$\begin{array}{llll}\text { H } & 4.881235000 & 0.331917000 & -2.841588000\end{array}$
H $\quad 7.268474000 \quad-1.249034000 \quad-3.719907000$
H $\quad 5.208767000 \quad-3.215709000 \quad-4.128825000$
$\begin{array}{llll}\text { H } & 4.182618000 & -0.978626000 & -0.935718000\end{array}$
$\begin{array}{llll}\text { H } & 6.445439000 & -3.291809000 & -2.837104000\end{array}$
H $\quad 5.773195000 \quad-0.917421000 \quad-4.608803000$
$\begin{array}{llll}\text { H } & 6.457342000 & 0.246356000 & -2.029254000\end{array}$
$\begin{array}{llll}\text { H } & 5.805019000 & -1.724202000 & -0.763713000\end{array}$
C $\quad-3.782261000 \quad-5.109525000 \quad-4.273533000$
C $\quad-4.016685000 \quad-5.967746000 \quad-3.018234000$ 


$\begin{array}{llll}\text { C } & -2.945375000 & -5.423930000 & -2.061958000 \\ \text { O } & -1.857059000 & -4.951784000 & -2.884908000 \\ \text { C } & -2.256023000 & -4.977357000 & -4.269869000 \\ \text { H } & -4.252198000 & -4.121204000 & -4.156178000 \\ \text { H } & -3.833974000 & -7.030518000 & -3.243171000 \\ \text { H } & -3.333872000 & -4.579937000 & -1.465041000 \\ \text { H } & -1.892686000 & -4.055956000 & -4.746602000 \\ \text { H } & -2.557236000 & -6.186051000 & -1.367818000 \\ \text { H } & -5.033962000 & -5.877489000 & -2.611800000 \\ \text { H } & -4.171085000 & -5.567759000 & -5.194150000 \\ \text { H } & -1.781686000 & -5.840743000 & -4.774885000 \\ \text { E(BPW91)=-1492.77496137 } & \end{array}$




$$
\left(C_{6} F_{5}\right) M g F
$$

$$
\begin{array}{lrrr}
\text { C } & -1.809215000 & -0.112780000 & -0.353224000 \\
\text { C } & -1.347827000 & -1.306283000 & 0.186371000 \\
\text { C } & -0.959703000 & 0.979537000 & -0.470390000 \\
\text { F } & -2.170701000 & -2.344777000 & 0.293225000 \\
\text { F } & -1.413162000 & 2.116530000 & -0.988645000 \\
\text { C } & -0.028090000 & -1.376271000 & 0.601083000 \\
\text { C } & 0.349301000 & 0.846521000 & -0.037574000 \\
\text { F } & 0.406775000 & -2.542436000 & 1.123928000 \\
\text { F } & 1.164118000 & 1.916389000 & -0.157111000 \\
\text { C } & 0.853278000 & -0.318292000 & 0.505124000 \\
\text { F } & 4.488477000 & -0.591867000 & 1.680535000 \\
\text { F } & -3.067899000 & -0.015617000 & -0.758785000 \\
\text { Mg } & 2.819998000 & -0.466667000 & 1.140405000
\end{array}
$$

$E(w B 97 X D)=-1027.77588827$ 


\section{Decaflurobiphenyl( $\left.C_{12} F_{10}\right)$}
C $\quad 0.078603000$
$-0.070288000$
$-3.940962000$
C -1.151294000
$-0.158037000$
$-3.298941000$
C $\quad 1.239933000$
0.10801000
$-3.197951000$
C -1.208481000
$-0.070126000$
$-1.914645000$
C $\quad 1.160133000$
0.200951000
$-1.815109000$
C $\quad-0.059918000$
0.11261400
$-1.142944000$
F $\quad-2.401793000$
$-0.176376000$
$-1.320273000$
F $\quad-2.264324000$
$-0.33549600$
$-4.013547000$
F
0.144315000
$-0.15706700$
$-5.268164000$
F 2.418959000
0.198398000
$-3.816595000$
F $\quad 2.289862000$
$0.391202000-1.125126000$
C -0.133029000
0.209328000
0.332793000
C $\quad-0.888269000$
1.203429000
0.956928000
C $\quad 0.550594000$
$-0.690019000$
1.152516000
C $\quad-0.966443000$
1.300687000
2.339558000
C $\quad 0.491633000$
$-0.60561700$
2.536961000
C $\quad-0.271736000$
0.392980000
3.130763000
F $\quad-1.69358800$
2.26252400
2.911505000
F $\quad-1.550993000$
2.100297000
0.218689000
F $\quad-0.337551000$
0.480073000
4.457934000
$\mathrm{F}$
1.15272900
$-1.479917000$
3.298244000
$\mathrm{F}$
$1.276894000-1.671274000$
0.606453000

$E(w B 97 X D)=-1455.5518491$ 
$\mathrm{MgF}_{2}$

\begin{tabular}{lrrr} 
Mg & 5.502405000 & 0.528322000 & 1.784321000 \\
$F$ & 5.502405000 & 0.528322000 & 0.014321000 \\
F & 5.502405000 & 0.528322000 & 3.554321000 \\
\multicolumn{2}{l}{ E(wB97XD) $=-399.9016379$} &
\end{tabular}




$$
\left(C_{6} F_{5}\right) M_{2} F_{3}(\text { single }- \text { bridge })
$$
$\begin{array}{llll}\text { C } & 3.336369441 & 0.006024140 & 0.003847402\end{array}$
$\begin{array}{llll}\text { C } & 2.647496055 & -1.185063863 & 0.191112519\end{array}$
$\begin{array}{llll}\text { C } & 2.643607539 & 1.194241823 & -0.187303649\end{array}$
F $\quad 4.663275752 \quad 0.008792148 \quad 0.007601231$
F $\quad 3.321758868 \quad-2.316881292 \quad 0.373218703$
$\begin{array}{llll}\text { C } & 1.262771304 & -1.153823492 & 0.181862368\end{array}$
$\begin{array}{llll}\text { F } & 3.314164841 & 2.328869998 & -0.365556924\end{array}$
$\begin{array}{llll}\text { C } & 1.258994597 & 1.157247973 & -0.185802022\end{array}$
$\begin{array}{llll}F & 0.605115079 & -2.320828737 & 0.365673059\end{array}$
$\begin{array}{llll}\text { C } & 0.531073630 & 0.000190916 & -0.004020473\end{array}$
F $\quad 0.597528370 \quad 2.321543772 \quad-0.373080761$
$\begin{array}{llll}\text { Mg } & -1.543985592 & -0.003933617 & -0.008368968\end{array}$
F $\quad-2.996839454 \quad-0.204011771 \quad-1.236259539$
F $\quad-3.001268805 \quad 0.190802428 \quad 1.215147621$
Mg $\quad-4.455072355 \quad-0.009241255 \quad-0.012674116$
F $\quad-6.216884813 \quad-0.012724172 \quad-0.015572455$

$E(w B 97 X D)=-1427.7767967$ 


\section{$\left(C_{6} F_{5}\right) M g_{2} F_{3}$ (bidentate)}
$\begin{array}{llll}\text { C } & 0.009055423 & 0.050365368 & 0.024169264\end{array}$
$\begin{array}{llll}\text { C } & 0.085137339 & 0.073504803 & 1.411979052\end{array}$
$\begin{array}{llll}\text { C } & 1.164818044 & -0.026870587 & -0.746826650\end{array}$
F $\quad-1.169072042 \quad 0.116379131 \quad-0.567351704$
$\begin{array}{llll}F & -1.009755637 & 0.177773246 & 2.145497355\end{array}$
$\begin{array}{llll}\text { C } & 1.347062323 & -0.007132817 & 1.963447633\end{array}$
F $\quad \begin{array}{lll}1.067179619 & -0.042842805 & -2.066261613\end{array}$
$\begin{array}{llll}\text { C } & 2.393218777 & -0.083341637 & -0.108396552\end{array}$
F $\quad \begin{array}{lll}1.461850700 & 0.093646036 & 3.344158618\end{array}$
$\begin{array}{llll}\text { C } & 2.534251870 & -0.086413155 & 1.269622133\end{array}$
$\begin{array}{llll}\text { F } & 3.494105616 & -0.168689897 & -0.870634304\end{array}$
$\begin{array}{llll}M g & 3.352866436 & 1.254764878 & 3.060042744\end{array}$
$\begin{array}{llll}\text { F } & 4.700481098 & -0.049189678 & 3.263158054\end{array}$
$\begin{array}{llll}\text { F } & 3.041599181 & 2.969514387 & 3.325212538\end{array}$
$\begin{array}{llll}\text { Mg } \quad 4.358125077 & -1.259562680 & 1.840392613\end{array}$
F $\quad 4.969946461 \quad-2.811578579 \quad 1.264408155$
$E(w B 97 X D)=-1427.7519095$ 


\section{Heterocoupling $\left(C_{6} F_{5}\right) M g F-\left(C_{6} F_{6}\right)(T S)$}
$\begin{array}{llll}\text { C } & -0.742447000 & 3.078690000 & 0.827907000\end{array}$
$\begin{array}{llll}\text { C } & -0.877083000 & 1.997338000 & 1.685012000\end{array}$
$\begin{array}{llll}\text { C } & 0.269618000 & 3.097302000 & -0.128709000\end{array}$
F $\quad-1.861607000 \quad 1.976643000 \quad 2.571581000$
F $\quad 0.364084000 \quad 4.122491000 \quad-0.963807000$
C $\quad 0.022516000 \quad 0.949067000 \quad 1.583650000$
$\begin{array}{llll}\text { C } & 1.145316000 & 2.034626000 & -0.189965000\end{array}$
F $\quad-0.163802000 \quad-0.098759000 \quad 2.394080000$
F $\quad 2.110273000 \quad 2.074682000 \quad-1.115861000$
$\begin{array}{llll}\text { C } & 1.071320000 & 0.931086000 & 0.663910000\end{array}$
F $\quad 0.578209000 \quad-2.375028000 \quad-1.107946000$
F $\quad-1.588510000 \quad 4.089012000 \quad 0.902826000$
$\begin{array}{llll}M g & 1.353722000 & -1.147505000 & -0.114486000\end{array}$
$\begin{array}{llll}\text { C } & 4.901395000 & 1.132266000 & 0.321107000\end{array}$
$\begin{array}{llll}\text { C } & 3.774971000 & 0.346709000 & 0.360878000\end{array}$
C $\quad 5.171184000 \quad 1.951418000 \quad 1.409521000$
F $\quad 3.403300000 \quad-0.447094000 \quad-0.698154000$
$\begin{array}{llll}F & 6.264294000 & 2.706801000 & 1.428027000\end{array}$
$\begin{array}{llll}\text { C } & 2.823395000 & 0.402445000 & 1.379212000\end{array}$
$\begin{array}{llll}\text { C } & 4.285096000 & 1.970268000 & 2.480311000\end{array}$
F $\quad 2.294588000 \quad-1.000492000 \quad 1.661630000$
F $\quad 4.535871000 \quad 2.755971000 \quad 3.517462000$
$\begin{array}{llll}\text { C } & 3.153319000 & 1.172657000 & 2.497414000\end{array}$
F $\quad 5.715216000 \quad 1.096070000 \quad-0.720329000$
F $\quad 2.297345000 \quad 1.245089000 \quad 3.515120000$
$E(w B 97 X D)=-1855.34077545$ 


$$
\left(C_{12} F_{9}\right) M g_{2} F_{3}(L M 1)
$$

$$
\begin{array}{lccc}
\text { C } & -0.529019000 & 3.477699000 & 0.780438000 \\
\text { C } & -0.643139000 & 2.433093000 & 1.679623000 \\
\text { C } & -0.353537000 & 3.176051000 & -0.566206000 \\
\text { F } & -0.680486000 & 2.642559000 & 3.014368000 \\
\text { F } & -0.231448000 & 4.163366000 & -1.441259000 \\
\text { C } & -0.600489000 & 1.141545000 & 1.204297000 \\
\text { C } & -0.258099000 & 1.845588000 & -0.957002000 \\
\text { F } & -0.678418000 & 0.174134000 & 2.208555000 \\
\text { F } & 0.017465000 & 1.610306000 & -2.250585000 \\
\text { C } & -0.364421000 & 0.771958000 & -0.090694000 \\
\text { F } & 0.438170000 & -2.733657000 & -1.330844000 \\
\text { F } & -0.560063000 & 4.732655000 & 1.192889000 \\
\text { Mg } & 0.451178000 & -1.134548000 & -0.587209000 \\
\text { C } & 3.744571000 & 1.659324000 & 0.348064000 \\
\text { C } & 3.006524000 & 0.490072000 & 0.320474000 \\
\text { C } & 3.745305000 & 2.414296000 & 1.516517000 \\
\text { F } & 2.846944000 & -0.225691000 & -0.815157000 \\
\text { F } & 4.437995000 & 3.542897000 & 1.558817000 \\
\text { C } & 2.321961000 & 0.111136000 & 1.453386000 \\
\text { C } & 2.977257000 & 1.994732000 & 2.596379000 \\
\text { F } & 1.577019000 & -1.058829000 & 1.290412000 \\
\text { F } & 2.927393000 & 2.812541000 & 3.660870000 \\
\text { C } & 2.223516000 & 0.834452000 & 2.609368000 \\
\text { F } & -0.185219000 & 0.187321000 & 5.483209000 \\
\text { F } & 4.412721000 & 2.063834000 & -0.717705000 \\
\text { Mg } & 0.582159000 & 0.596488000 & 3.948960000 \\
\text { E(wB97XD)=-2055.59673906} & \\
\hline & &
\end{array}
$$




$$
\left(C_{12} F_{9}\right) M g_{2} F_{3}(L M 2)
$$

$$
\begin{array}{llll}
\text { C } & -4.092657000 & 1.634421000 & 0.236027000 \\
\text { C } & -2.913653000 & 2.173819000 & -0.257040000 \\
\text { C } & -4.239526000 & 0.254605000 & 0.316276000 \\
\text { F } & -2.742805000 & 3.487685000 & -0.328366000 \\
\text { F } & -5.368146000 & -0.251578000 & 0.793551000 \\
\text { C } & -1.939491000 & 1.277659000 & -0.649747000 \\
\text { C } & -3.197415000 & -0.562342000 & -0.098613000 \\
\text { F } & -0.750326000 & 1.895378000 & -1.138431000 \\
\text { F } & -3.377940000 & -1.888473000 & 0.010069000 \\
\text { C } & -2.000588000 & -0.085314000 & -0.605685000 \\
\text { F } & -0.406270000 & -3.141583000 & -1.933994000 \\
\text { F } & -5.071632000 & 2.433032000 & 0.633540000 \\
\text { Mg } & -0.508048000 & -1.516590000 & -1.240561000 \\
\text { C } & 3.046711000 & 4.745156000 & 2.244267000 \\
\text { C } & 3.642710000 & 4.788353000 & 0.987968000 \\
\text { C } & 2.053935000 & 3.813824000 & 2.523211000 \\
\text { F } & 4.588194000 & 5.691055000 & 0.751739000 \\
\text { F } & 1.483466000 & 3.769422000 & 3.720568000 \\
\text { C } & 3.240597000 & 3.892773000 & 0.005604000 \\
\text { C } & 1.712247000 & 2.960273000 & 1.495088000 \\
\text { F } & 3.836617000 & 3.970465000 & -1.190195000 \\
\text { F } & 0.726467000 & 2.002199000 & 1.710070000 \\
\text { C } & 2.260853000 & 2.951672000 & 0.237931000 \\
\text { F } & 1.025995000 & -0.372439000 & -1.019469000 \\
\text { F } & 3.429701000 & 5.599626000 & 3.182644000 \\
\text { Mg } & 1.131948000 & 1.418272000 & -0.569562000 \\
\text { E(wB97XD)=-2055.62519990} & \\
\hline & &
\end{array}
$$




$$
\left(C_{12} F_{9}\right) M g_{2} F_{3}(L M 3)
$$

$$
\begin{array}{lccc}
\text { C } & -0.393437000 & 2.609081000 & -0.120361000 \\
\text { C } & -0.018174000 & 2.366214000 & 1.194386000 \\
\text { C } & 0.243283000 & 1.950968000 & -1.164261000 \\
\text { F } & -1.365238000 & 3.474262000 & -0.380443000 \\
\text { F } & -0.635280000 & 3.002364000 & 2.185988000 \\
\text { C } & 0.999716000 & 1.457377000 & 1.432019000 \\
\text { F } & -0.124663000 & 2.191480000 & -2.419474000 \\
\text { C } & 1.253665000 & 1.054030000 & -0.859027000 \\
\text { F } & 1.354384000 & 1.230597000 & 2.716836000 \\
\text { C } & 1.661340000 & 0.779741000 & 0.429550000 \\
\text { F } & 1.864624000 & 0.420285000 & -1.885293000 \\
\text { Mg } & 3.178450000 & -0.576957000 & 0.835112000 \\
\text { F } & 5.079652000 & -0.618332000 & 1.059264000 \\
\text { C } & 8.876531000 & -5.673554000 & 2.357216000 \\
\text { C } & 8.499093000 & -5.431438000 & 1.042958000 \\
\text { C } & 8.241282000 & -5.015125000 & 3.401818000 \\
\text { F } & 9.849006000 & -6.538314000 & 2.616176000 \\
\text { F } & 9.114777000 & -6.067853000 & 0.050651000 \\
\text { C } & 7.480556000 & -4.523002000 & 0.806593000 \\
\text { F } & 8.611344000 & -5.254933000 & 4.656546000 \\
\text { C } & 7.230128000 & -4.118618000 & 3.097847000 \\
\text { F } & 3.401449000 & -2.447478000 & 1.180328000 \\
\text { C } & 6.820325000 & -3.845077000 & 1.809776000 \\
\text { F } & 6.620722000 & -3.484606000 & 4.124800000 \\
\text { Mg } & 5.302700000 & -2.488906000 & 1.404380000 \\
\text { F } & 7.123726000 & -4.296851000 & -0.477778000 \\
\text { E(wB97XD)=-2055.65017265} & \\
\hline & &
\end{array}
$$




$$
\left(C_{12} F_{9}\right) M g_{2} F_{3}(T S 1)
$$

$$
\begin{aligned}
& \begin{array}{llll}
\text { C } & 0.109570000 & -0.987243000 & -0.541786000
\end{array} \\
& \text { C } \quad 0.183357000 \quad 0.305873000 \quad-1.046459000 \\
& \begin{array}{llll}
\text { C } & 1.107289000 & -1.460396000 & 0.299392000
\end{array} \\
& \text { F } \quad-0.909688000 \quad-1.769362000 \quad-0.863354000 \\
& \text { F } \quad-0.773710000 \quad 0.746551000 \quad-1.851073000 \\
& \begin{array}{llll}
\text { C } & 1.262787000 & 1.103311000 & -0.696302000
\end{array} \\
& \begin{array}{llll}
\text { F } & 1.054941000 & -2.692051000 & 0.786865000
\end{array} \\
& \begin{array}{llll}
\text { C } & 2.139120000 & -0.588784000 & 0.587654000
\end{array} \\
& \text { F } \quad 1.304947000 \quad 2.347981000 \quad-1.208365000 \\
& \begin{array}{llll}
\text { C } & 2.284754000 & 0.690802000 & 0.137954000
\end{array} \\
& \text { F } \quad 3.127436000 \quad-1.132292000 \quad 1.460104000 \\
& \begin{array}{llll}
M g & 5.097603000 & -0.884919000 & 1.821203000
\end{array} \\
& \text { F } \quad 5.954198000 \quad-2.216402000 \quad 2.579638000 \\
& \begin{array}{llll}
\text { F } & 5.233675000 & 0.770963000 & 1.014140000
\end{array} \\
& \begin{array}{llll}
M g & 3.852907000 & 2.062095000 & 0.662182000
\end{array} \\
& \begin{array}{llll}
\text { C } & 4.067266000 \quad 3.886089000 & 1.659564000
\end{array} \\
& \text { C } 4.096302000 \quad 3.695411000 \quad 3.022472000 \\
& \text { C } \quad 4.229310000 \quad 4.709756000 \quad 3.953233000 \\
& \begin{array}{llll}
\text { C } & 4.344666000 & 6.008918000 & 3.477480000
\end{array} \\
& \begin{array}{llll}
\text { C } & 4.323397000 & 6.258769000 & 2.111295000
\end{array} \\
& \begin{array}{llll}
\text { F } & 4.475533000 & 7.017094000 & 4.332140000
\end{array} \\
& \text { F } \quad 4.248997000 \quad 4.474148000 \quad 5.263005000 \\
& \text { F } \quad 4.436957000 \quad 7.510300000 \quad 1.673195000 \\
& \begin{array}{llll}
\text { C } & 4.186083000 & 5.192024000 & 1.235246000
\end{array} \\
& \text { F } \quad 3.977607000 \quad 2.414046000 \quad 3.492441000 \\
& \begin{array}{llll}
\text { F } & 4.174213000 & 5.468547000 & -0.082220000
\end{array} \\
& E(w B 97 X D)=-2055.62459994
\end{aligned}
$$




$$
\left(C_{12} F_{9}\right) M g_{2} F_{3}(T S 2)
$$

$$
\begin{array}{lrrr}
\text { C } & -0.105891000 & -0.290604000 & 0.060965000 \\
\text { C } & -0.065459000 & -0.303576000 & 1.445632000 \\
\text { C } & 1.067203000 & -0.145593000 & -0.667061000 \\
\text { F } & -1.266481000 & -0.403602000 & -0.568217000 \\
\text { F } & -1.179434000 & -0.415490000 & 2.156150000 \\
\text { C } & 1.178168000 & -0.182125000 & 2.027400000 \\
\text { F } & 1.012721000 & -0.129655000 & -1.992806000 \\
\text { C } & 2.266057000 & -0.018132000 & 0.010695000 \\
\text { F } & 1.194159000 & -0.137201000 & 3.441395000 \\
\text { C } & 2.377287000 & -0.041653000 & 1.388402000 \\
\text { F } & 3.384432000 & 0.116743000 & -0.728945000 \\
\text { Mg } & 2.633775000 & 1.238433000 & 3.864326000 \\
\text { Mg } & 4.465073000 & -0.469493000 & 1.991965000
\end{array}
$$$$
\text { F } \quad 5.546787000 \quad-1.770085000 \quad 1.491173000
$$$$
\begin{array}{llll}
\text { F } & 4.234217000 & 0.311559000 & 3.722335000
\end{array}
$$$$
\text { F } \quad 1.987428000 \quad 2.626784000 \quad 4.743542000
$$$$
\begin{array}{llll}
\text { C } & 5.771121000 & 3.580101000 & 2.195125000
\end{array}
$$$$
\begin{array}{llll}
\text { C } & 4.892881000 & 2.597113000 & 1.772560000
\end{array}
$$$$
\begin{array}{llll}
\text { C } & 5.290452000 & 4.777924000 & 2.763016000
\end{array}
$$$$
\text { F } \quad 7.078306000 \quad 3.414240000 \quad 2.095270000
$$$$
\begin{array}{llll}
F & 5.363699000 & 1.407370000 & 1.322506000
\end{array}
$$$$
\begin{array}{llll}
\text { C } & 3.571651000 & 2.876794000 & 1.997366000
\end{array}
$$$$
\text { F } \quad 6.175437000 \quad 5.699272000 \quad 3.105910000
$$$$
\begin{array}{llll}
\text { C } \quad 3.931347000 & 5.013685000 & 2.969203000
\end{array}
$$$$
\begin{array}{llll}
\text { C } \quad 3.210375000 & 3.972073000 & 2.457088000
\end{array}
$$$$
\begin{array}{llll}
\text { F } & 3.491739000 & 6.124519000 & 3.509536000
\end{array}
$$

$$
E(w B 97 X)=-2055.570249
$$

$$
\left(C_{12} F_{9}\right) M g_{2} F_{3}(T S 3)
$$




\begin{tabular}{|c|c|c|c|}
\hline C & 0.921699000 & 1.767718000 & 4.222400000 \\
\hline C & 2.261466000 & 1.838329000 & 4.578718000 \\
\hline C & 0.523331000 & 0.975243000 & 3.154755000 \\
\hline $\mathrm{F}$ & 0.018126000 & 2.461954000 & 4.904373000 \\
\hline $\mathrm{F}$ & 2.632115000 & 2.602179000 & 5.603944000 \\
\hline C & 3.180733000 & 1.103799000 & 3.847102000 \\
\hline $\mathrm{F}$ & -0.763550000 & 0.915323000 & 2.820016000 \\
\hline C & 1.491605000 & 0.264937000 & 2.464262000 \\
\hline $\mathrm{F}$ & 4.478192000 & 1.190495000 & 4.210417000 \\
\hline C & 2.833862000 & 0.300867000 & 2.780128000 \\
\hline $\mathrm{F}$ & 1.084970000 & -0.498529000 & 1.425055000 \\
\hline $\mathrm{Mg}$ & 4.220264000 & -0.814924000 & 1.698984000 \\
\hline $\mathrm{F}$ & 5.700218000 & -0.494336000 & 0.514225000 \\
\hline C & 6.809830000 & -5.261636000 & 3.006127000 \\
\hline C & 7.545911000 & -5.098965000 & 1.830509000 \\
\hline C & 6.203057000 & -4.175830000 & 3.678788000 \\
\hline $\mathrm{F}$ & 6.640314000 & -6.473518000 & 3.506287000 \\
\hline $\mathrm{F}$ & 8.059717000 & -6.107327000 & 1.175247000 \\
\hline C & 7.670741000 & -3.759249000 & 1.577260000 \\
\hline $\mathrm{F}$ & 5.427177000 & -4.442371000 & 4.715040000 \\
\hline C & 6.379566000 & -2.876081000 & 3.231856000 \\
\hline $\mathrm{F}$ & 4.355851000 & -2.567083000 & 0.922647000 \\
\hline C & 7.150061000 & -2.748582000 & 2.101715000 \\
\hline $\mathrm{F}$ & 5.655357000 & -1.865027000 & 3.742605000 \\
\hline $\mathrm{Mg}$ & 6.035591000 & -2.312721000 & 0.016839000 \\
\hline$F$ & 7.221247000 & -3.442137000 & -0.744202000 \\
\hline
\end{tabular}

$$
\left(C_{12} F_{9}\right) M g_{2} F_{3}(T S 4)
$$




\begin{tabular}{lccc} 
C & -0.150767000 & -0.457571000 & 0.173701000 \\
C & 0.030746000 & 0.637093000 & 1.007199000 \\
C & 0.922869000 & -1.278794000 & -0.142078000 \\
F & -1.354046000 & -0.720705000 & -0.321474000 \\
F & -1.002851000 & 1.420594000 & 1.306519000 \\
C & 1.299312000 & 0.881615000 & 1.506233000 \\
F & 0.738903000 & -2.326352000 & -0.941506000 \\
C & 2.166327000 & -0.977543000 & 0.390315000 \\
F & 1.442796000 & 1.957859000 & 2.319707000 \\
C & 2.399121000 & 0.097922000 & 1.223246000 \\
F & 3.194190000 & -1.790346000 & 0.065924000 \\
Mg & 4.283578000 & 0.570340000 & 1.990237000 \\
F & 6.080043000 & -0.093474000 & 1.999640000 \\
F & 4.810937000 & 0.887597000 & 3.933641000 \\
Mg & 6.627779000 & 0.243913000 & 3.782483000 \\
F & 8.016993000 & 0.140246000 & 4.860179000 \\
C & 3.149825000 & 4.858237000 & 2.229040000 \\
C & 3.910307000 & 4.034111000 & 1.410677000 \\
C & 3.044938000 & 4.630054000 & 3.618269000 \\
F & 2.488848000 & 5.886546000 & 1.724791000 \\
F & 3.938971000 & 4.236598000 & 0.104776000 \\
C & 4.506183000 & 2.950554000 & 2.038077000 \\
F & 2.355102000 & 5.490878000 & 4.345113000 \\
C & 3.664843000 & 3.548333000 & 4.242248000 \\
C & 4.401519000 & 2.893673000 & 3.295317000 \\
F & 3.591427000 & 3.319174000 & 5.529897000 \\
\hline
\end{tabular}

$\left(C_{12} F_{9}\right) M g_{2} F_{3}(P 1)$ 


\begin{tabular}{lrrr} 
C & -3.639485000 & -0.554687000 & 0.266313000 \\
C & -2.802044000 & -1.089224000 & -0.703392000 \\
C & -3.178082000 & 0.447565000 & 1.106972000 \\
F & -3.228138000 & -2.046636000 & -1.508884000 \\
F & -3.975701000 & 0.940873000 & 2.036181000 \\
C & -1.518176000 & -0.592139000 & -0.806078000 \\
C & -1.878993000 & 0.915647000 & 0.977819000 \\
F & -0.717918000 & -1.130381000 & -1.775841000 \\
F & -1.453718000 & 1.844434000 & 1.818315000 \\
C & -1.013198000 & 0.406523000 & 0.014958000 \\
F & 2.179269000 & -1.844615000 & -2.967250000 \\
F & -4.873243000 & -1.006636000 & 0.389557000 \\
Mg & 1.312419000 & -1.851185000 & -1.427801000 \\
C & 2.063769000 & 2.553743000 & -0.335585000 \\
C & 0.731618000 & 2.151309000 & -0.305144000 \\
C & 3.072759000 & 1.629204000 & -0.125672000 \\
F & -0.208257000 & 3.067085000 & -0.520722000 \\
F & 4.337225000 & 2.014659000 & -0.153667000 \\
C & 0.409015000 & 0.828919000 & -0.062714000 \\
C & 2.718436000 & 0.307667000 & 0.114379000 \\
F & 0.784875000 & -3.025449000 & -0.025946000 \\
F & 3.717394000 & -0.572851000 & 0.313431000 \\
C & 1.414152000 & -0.142646000 & 0.149284000 \\
F & -0.233763000 & -1.689954000 & 2.929844000 \\
\hline & 2.366366000 & 3.818207000 & -0.569854000 \\
\hline & 0.696392000 & -1.810849000 & 1.428641000 \\
\hline
\end{tabular}

$\left(C_{12} F_{9}\right) M g_{2} F_{3}(P 2)$ 


$$
\begin{aligned}
& \begin{array}{llll}
\text { C } & -1.194807000 & -2.981131000 & -1.463749000
\end{array} \\
& \text { C } \quad-1.546916000 \quad-2.276171000 \quad-0.324330000 \\
& \text { C } \quad-0.411335000 \quad-2.365939000 \quad-2.427428000 \\
& \text { F } \quad-1.585769000 \quad-4.234505000 \quad-1.619134000 \\
& \text { F } \quad-2.261321000 \quad-2.850505000 \quad 0.634335000 \\
& \begin{array}{llll}
\text { C } & -1.126596000 & -0.967947000 & -0.196168000
\end{array} \\
& \text { F } \quad-0.046654000 \quad-3.043580000 \quad-3.504567000 \\
& \begin{array}{llll}
\text { C } & 0.015064000 & -1.057360000 & -2.250689000
\end{array} \\
& \text { F } \quad-1.474574000 \quad-0.338952000 \quad 0.969118000 \\
& \text { C } \quad-0.348274000 \quad-0.302064000 \quad-1.135883000 \\
& \text { F } \quad 0.822128000 \quad-0.547171000 \quad-3.170253000 \\
& \begin{array}{llll}
M g & 0.108860000 & -1.617756000 & 4.205779000
\end{array} \\
& \begin{array}{llll}
M g & 0.407349000 & 0.269102000 & 2.022539000
\end{array} \\
& \text { F } \quad 0.822539000 \quad-1.565324000 \quad 2.435585000 \\
& \text { F } \quad-0.214991000 \quad 0.229856000 \quad 3.830073000 \\
& \text { F } \quad-0.091772000 \quad-2.758234000 \quad 5.535258000 \\
& \begin{array}{llll}
\text { C } & 0.548231000 & 3.300302000 & -1.788962000
\end{array} \\
& \begin{array}{llll}
\text { C } & 0.046069000 & 2.015931000 & -1.948560000
\end{array} \\
& \begin{array}{llll}
\text { C } & 1.124557000 & 3.668844000 & -0.585214000
\end{array} \\
& \text { F } \quad 0.463729000 \quad 4.177346000 \quad-2.782331000 \\
& \text { F } \quad-0.546541000 \quad 1.720909000 \quad-3.107334000 \\
& \begin{array}{llll}
\text { C } & 0.141008000 & 1.084637000 & -0.921097000
\end{array} \\
& \text { F } \quad 1.598638000 \quad 4.898493000 \quad-0.429315000 \\
& \begin{array}{llll}
\text { C } & 1.166749000 & 2.734189000 & 0.439696000
\end{array} \\
& \begin{array}{llll}
\text { C } & 0.694406000 & 1.449865000 & 0.321252000
\end{array} \\
& \text { F } \quad 1.686246000 \quad 3.136972000 \quad 1.623123000 \\
& E(w B 97 X D)=-2055.7302271
\end{aligned}
$$

